\title{
On Generalized Interval Valued Fuzzy Soft Matrices
}

\author{
Fazal Dayan*, Muhammad Zulqarnain \\ Department of Mathematics, University of Management and Technology, Lahore, Pakistan
}

Email address:

fazaldayan1@gmail.com (F. Dayan)

${ }^{*}$ Corresponding author

\section{To cite this article:}

Fazal Dayan, Muhammad Zulqarnain. On Generalized Interval Valued Fuzzy Soft Matrices. American Journal of Mathematical and Computer Modelling. Vol. x, No. x, 2018, pp. x-x. doi: 10.11648/j.ajmcm.20180301.11

Received: February 16, 2018; Accepted: March 9, 2018; Published: March 30, 2018

\begin{abstract}
Interval valued fuzzy soft set was a combination of the interval valued fuzzy set and soft set, while in generalized interval valued fuzzy soft set a degree was attached with the parameterization of fuzzy sets in defining an interval valued fuzzy soft set. In this paper we introduced the concept of generalized interval valued fuzzy soft matrices. We discussed some of its types and some operations. We also discussed about the similarity of two generalized interval valued fuzzy soft matrices.
\end{abstract}

Keywords: Interval Valued Fuzzy Soft Set (IVFSS), Generalized Fuzzy Soft Set, Generalized IVFSS, Similarity

\section{Introduction}

A lot of problems in our real life in economics, social sciences, medical sciences, environmental sciences and engineering etc. involve various uncertainties. Many theories have been developed to deal with these uncertainties. Some of these theories are probability theory [1], fuzzy set theory (FST) [2], rough set theory (RST) [3], interval mathematics [4] and intuitionistic fuzzy set theory (IFST) [5] etc. Molodtsov [6] pointed out that all these theories have some inherent difficulties. He proposed soft set theory (SST) to overcome these difficulties. It was a generic mathematical tool for dealing problems having uncertainty. Later Maji and Biswas [7] defined soft subset and soft super set. They also defined absolute soft set and null soft set. They introduced some operations on soft set and De Morgan's laws are also verified by them. Ali et al [8] pointed out some errors of the previous work and introduced some new operations. They further studied more and discussed some algebraic structures of soft sets. Maji et al. [9] proposed fuzzy soft set (FSS), an improvement of the SST by combining (FST) and (SST). Roy and Maji [10] gave an application of fuzzy soft set in decision making. Yang et al. [11] introduced the intervalvalued fuzzy soft set (IVFSS) which was a combination of the IVFS and SST.

Majumdar and Samanta [12] introduced the concept of generalized fuzzy soft sets (GFSS). B. K. Saikia et al. [13] defined generalized fuzzy soft matrix (GFSM) and applied it to a decision making (DM) problem. Shawkat Alkhazaleh and Abdul Razak Salleh [14] introduced generalized interval valued fuzzy soft set (GIVFSS). In their generalization of FSS, they attached a degree with the parameterization of fuzzy sets in defining an IVFSS. They discussed various operations and properties of GIVFSS. Some of these are GIVFS subset, GIVFS equal set, generalized null interval valued fuzzy soft set (GNIVFS), generalized absolute interval valued fuzzy soft set (GAIVFS), compliment of GIVFSS, union of GIVFSS's and intersection of GIVFSS's. They defined AND and OR operations on GIVFSS and similarity measure of two GIVFSS's. They also give some applications of GIVFSS in DM problem and medical diagnosis.

Mi Jung Son [15] introduced interval valued fuzzy soft set and defined some of its types. P. Rajarajeswari and P. Dhanalakshmi [16] developed interval-valued fuzzy soft matrix theory. Zulqarnain. M and M. Saeed [17] defined some new types of interval valued fuzzy soft matrix and gave an application of IVFSM in a decision making problem. Anjan Mukherjee and Sadhan Sarkar [18, 19] introduced Similarity measures for interval-valued intuitionistic fuzzy soft sets and gave applications in medical diagnosis problems. B. Chetia and P. K. Das [20] used interval-valued fuzzy soft sets and Sanchez's approach for medical diagnosis. In recent years many researchers [21-25] have been worked on applications of interval valued fuzzy soft sets.

In this paper we extended the concept of GIVFSS and introduced generalized interval valued fuzzy soft matrix (GIVFSM). We defined different types of GIVFSM's and 
studied some properties. We also discussed some operators on the basis of weights and some of their properties.

\section{Some Basic Definitions}

Definition 2.1. [1] Let $X$ be a universal set, $P$ be set of parameters and $\mathrm{I}^{\mathrm{X}}$ be the set of all fuzzy subsets of $\mathrm{X}$. Let $\mathrm{A} \subseteq \mathrm{P}$. A pair $(\mathrm{F}, \mathrm{A})$ is a fuzzy soft set over $\mathrm{X}$, where

$\mathrm{F}: \mathrm{A} \rightarrow \mathrm{I}^{\mathrm{X}}$.

Definition 2. 2. [11] An IVFSS $X$ on a universe $U$ is a mapping such that

$X: U \rightarrow \operatorname{Int}([0,1])$, where $\operatorname{Int}([0,1])$ represents the set of all closed subintervals of $[0,1]$, the set of all interval valued fuzzy sets on $U$ is denoted by $P(U)$. Suppose that $X \in P(U)$, $\forall x \in U$,

$\mu_{x}(x)=\left[\mu^{-}{ }_{x}(x), \mu^{+}{ }_{x}(x)\right]$ is the degree of membership $x$ to $X$, where $\mu^{-}{ }_{x}(x)$ and $\mu^{+}{ }_{x}(x)$ are the lower and upper degrees of membership of $x$ to $X$ respectively, such that

$$
0 \leq \mu^{-}{ }_{x}(x) \leq \mu^{+}{ }_{x}(x) \leq 1
$$

Definition 2. 3. [26] Let $X$ be the universal set and $P$ be the set of parameters. Suppose that $\mathrm{A} \subseteq \mathrm{P}$ and $(\mathrm{F}, \mathrm{A})$ be a fuzzy soft set. Then the matrix form of the fuzzy soft set $(F, A)$ is given as

$$
A=\left[a_{i j}\right]_{m \times n}, i=1,2,3, \ldots, m \text { and } j=1,2,3, \ldots, n
$$

where

$$
a_{i j}=\left\{\begin{array}{r}
u_{j}\left(p_{i}\right), p_{j} \in A \\
0, p_{j} \notin A
\end{array}\right.
$$

$\forall \mathrm{i}, \mathrm{j}$.

Here $\mu_{j}\left(p_{i}\right)$ denotes the membership of $\mathrm{P}_{\mathrm{i}}$ in the fuzzy soft set $\mathrm{F}\left(\mathrm{p}_{\mathrm{j}}\right)$.

Definition 2. 4. [13] Let $X$ be the universal set, $P$ be the set of parameters and $A \subseteq P$. Let $\left(F_{\lambda}, P\right)$ be a GFSS over $(X, P)$. A subset of $\mathrm{X} \times \mathrm{P}, \mathrm{R}_{\mathrm{A}}=\left\{(\mathrm{x}, \mathrm{p}), \mathrm{p} \in \mathrm{P}, \mathrm{x} \in \mathrm{F}_{\lambda}(\mathrm{p})\right\}$ is a relation form of $\left(\mathrm{F}_{\lambda}, \mathrm{P}\right)$, where

$$
\mu R_{A}: \mathrm{X} \times \mathrm{P} \rightarrow[0,1] \text { and } \lambda \mathrm{R}_{\mathrm{A}}: \mathrm{X} \times \mathrm{P} \rightarrow[0,1],
$$

such that

$$
R_{A}:(\mathrm{x}, \mathrm{p}) \in[0,1], \forall \mathrm{x} \in \mathrm{X}, \mathrm{p} \in \mathrm{P}
$$

and

$\lambda \mathrm{R}_{\mathrm{A}}:(\mathrm{x}, \mathrm{p}) \in[0,1], \forall \mathrm{x} \in \mathrm{X}, \mathrm{p} \in \mathrm{P}$.

If $\left[\mu_{\mathrm{ij}}, \lambda_{\mathrm{j}}\right]_{\mathrm{m} \times \mathrm{n}}=\mu R_{A}\left(\left(\mathrm{x}_{\mathrm{i}}, \mathrm{p}_{\mathrm{j}}\right), \lambda\left(\mathrm{x}_{\mathrm{i}}, \mathrm{p}_{\mathrm{j}}\right)\right)$, then we can define an $\mathrm{m} \times \mathrm{n}$ generalized fuzzy soft matrix (GFSM) of GFSS over $(\mathrm{X}, \mathrm{P})$ as

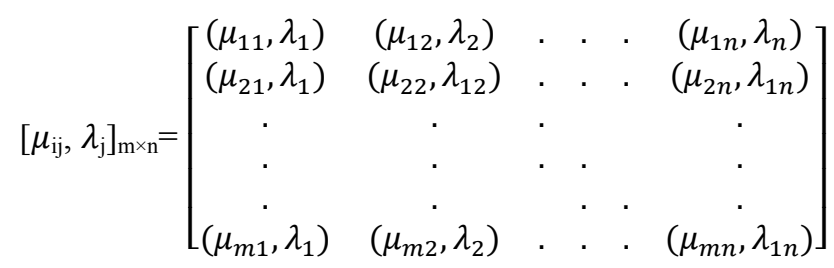

Definition 2. 5. [16] Let $\mathrm{U}=\left\{c_{1}, c_{2}, c_{3} \ldots, c_{m}\right\}$ be the Universe set and $\mathrm{E}$ be the set of parameters given by $\mathrm{E}=$ $\left\{e_{1}, e_{2}, e_{3} \ldots, e_{n}\right\}$. Let $\mathrm{A}$ be a subset of $\mathrm{E}$ and $(\mathrm{F}, \mathrm{A})$ be an interval valued fuzzy soft set over $\mathrm{U}$ and $\mathrm{F}$ is a mapping given by $F$ : $A \rightarrow I^{\mathrm{U}}$, where $\mathrm{I}^{\mathrm{U}}$ denotes the collection of all Interval valued fuzzy subsets of $U$. Then the Interval valued fuzzy soft set can expressed in matrix form as

$$
\mathrm{A}=\left[\mathrm{a}_{\mathrm{ij}}\right]_{\mathrm{mxn}} \text { or } \tilde{\mathrm{A}}=\left[\mathrm{a}_{\mathrm{ij}}\right] \mathrm{i}=1,2, \ldots, \mathrm{m}, \mathrm{j}=1,2, \ldots, \mathrm{n} .
$$

Where

$$
a_{i j}=\left\{\begin{array}{c}
\mu_{j L\left(C_{i}\right)}, \text { if } e_{j} \in A \\
{[0,0], \text { if } e_{j} \notin A}
\end{array}\right.
$$

The interval $\left[\mu_{\mathrm{jL}}\left(\mathrm{c}_{\mathrm{i}}\right), \mu_{\mathrm{ju}}\left(\mathrm{c}_{\mathrm{i}}\right)\right]$ represents the membership of $c_{i}$ in the Interval valued fuzzy set $F\left(e_{j}\right)$.

If $\mu_{\mathrm{jL}}\left(\mathrm{c}_{\mathrm{i}}\right)=\mu_{\mathrm{ju}}\left(\mathrm{c}_{\mathrm{i}}\right)$ then the Interval- valued fuzzy soft matrix (IVFSM) reduces to a FSM.

Definition 2.6. [11] Let $U$ be an initial Universe set and E be the set of parameters, let

$A \subseteq$ E. A pair $(F, A)$ is called Interval valued fuzzy soft set over $U$ where $F$ is a mapping given by $F$ : $A \rightarrow I^{U}$, where $I^{U}$ denotes the collection of all Interval valued fuzzy subsets of U.

Definition 2.7. [14] Let $U$ be the Universal set and $E$ be the set of parameters. Let $\mathrm{A} \subseteq \mathrm{E}$ and $\mu$ be a fuzzy subset of $\mathrm{A}$. Let $\mathrm{F}: \mathrm{A} \rightarrow \mathrm{P}(\mathrm{U})$ and $\mu: \mathrm{A} \rightarrow \mathrm{I}=[0,1]$ where $\mathrm{P}(\mathrm{U})$ is the collection of all Interval valued fuzzy subsets of $U$.

Define a function $\mathrm{F}_{\mu}: \mathrm{A} \rightarrow \mathrm{P}(\mathrm{U}) \times \mathrm{I}$, such that

$$
\begin{gathered}
\mathrm{F}_{\mu}(\mathrm{e})=\mathrm{F}(\mathrm{e})=(F(e), \mu(e)) \\
\mathrm{F}_{\mu}\left(e_{i}\right)=\left(F\left(e_{i}\right)(\mathrm{x}), \mu\left(e_{i}\right)\right), \forall\left(e_{i}\right) .
\end{gathered}
$$

where $F\left(e_{i}\right)(\mathrm{x})$ is an interval value and is called degree of membership of an element $\mathrm{x}$ to $\mathrm{F}(\mathrm{e})$ and $\mu(e)$ is called the degree of possibility of this belongness. Then $F_{\mu}$ is a GIVFSS.

\section{Generalized Interval Valued Fuzzy Soft Matrices}

Definition 3.1 Let $U$ be the Universal set and $E$ be the set of parameters. Let $\mathrm{A} \subseteq \mathrm{E}$ and $\mu$ be a fuzzy subset of $\mathrm{A}$. Let $\mathrm{F}$ : $\mathrm{A} \rightarrow \mathrm{P}(\mathrm{U})$ and $\mu: \mathrm{A} \rightarrow \mathrm{I}=[0,1]$ where $\mathrm{P}(\mathrm{U})$ is the collection of all Interval valued fuzzy subsets of U. A function $\mathrm{F}_{\mu}: \mathrm{A} \rightarrow \mathrm{P}(\mathrm{U}) \times \mathrm{I}$ defined as

$$
\begin{gathered}
\mathrm{F}_{\mu}(\mathrm{e})=\mathrm{F}(\mathrm{e})=(F(e), \mu(e)), \text { where } \mathrm{F}_{\mu}\left(e_{j}\right)= \\
\left(F\left(e_{j}\right)\left(\mathrm{x}_{\mathrm{i}}\right), \mu\left(e_{i}\right)\right), \forall\left(e_{j}\right) .
\end{gathered}
$$

Then the generalized interval valued fuzzy soft set $\mathrm{F}_{\mu}$ can be expressed in matrix form as

$$
\left(\mathrm{F}_{\mu}\left(e_{j}\right), \mu\right)=\left[\mathrm{a}_{\mathrm{ij}}\right]_{\mathrm{m} \times \mathrm{n}}
$$

where

$$
=\left\{\begin{array}{r}
\left(F\left(e_{j}\right)\left(x_{i}\right), \mu\left(e_{j}\right)\right), \text { if } \mathrm{e}_{\mathrm{j}} \in \mathrm{A} \text { and } \mathrm{i}=1,2, \ldots, \mathrm{m}, j=1,2, \ldots, n \\
(0,0), \text { if } e_{j} \in A \text { and } \mathrm{i}=1,2, \ldots, \mathrm{m}, j=1,2, \ldots, n
\end{array}\right.
$$$$
\text { i.e. }\left[a_{i j}\right]_{m \times n}=
$$ 


$\left[\begin{array}{cccc}\left(\mathrm{F}\left(\mathrm{e}_{1}\right)\left(\mathrm{x}_{1}\right), \mu\left(\mathrm{e}_{1}\right)\right) & \left(\mathrm{F}\left(\mathrm{e}_{1}\right)\left(\mathrm{x}_{2}\right), \mu\left(\mathrm{e}_{1}\right)\right) & \cdot & \left(\mathrm{F}\left(\mathrm{e}_{1}\right)\left(\mathrm{x}_{\mathrm{m}}\right), \mu\left(\mathrm{e}_{1}\right)\right) \\ & \left(\mathrm{F}\left(\mathrm{e}_{2}\right)\left(\mathrm{x}_{1}\right), \mu\left(\mathrm{e}_{2}\right)\right) & \\ \cdot & \cdot & \cdot & \cdot \\ \left(\mathrm{F}\left(\mathrm{e}_{\mathrm{n}}\right)\left(\mathrm{x}_{1}\right), \mu\left(\mathrm{e}_{\mathrm{n}}\right)\right) & \cdot & \cdot & \left(\mathrm{F}\left(\mathrm{e}_{\mathrm{n}}\right)\left(\mathrm{x}_{\mathrm{m}}\right), \mu\left(\mathrm{e}_{\mathrm{n}}\right)\right)\end{array}\right]$

where $F\left(e_{j}\right)\left(x_{i}\right)=\left[F\left(e_{j L}\right)\left(x_{i}\right), F\left(e_{j U}\right)\left(x_{i}\right)\right]$ represents the membership of $e_{j}$ in the GIVFSS $\mathrm{F}_{\mu}\left(e_{j}\right)$, such that

$$
0 \leq F\left(e_{j L}\right)\left(x_{i}\right) \leq F\left(e_{j U}\right)\left(x_{i}\right) \leq 1 .
$$

If $F\left(e_{j}\right)\left(x_{i}\right)=\mu\left(e_{j}\right)$ then the GIVFSM reduces to a GFSM

Definition 3.2. Let $\mathrm{F}_{\mu}$ and $\mathrm{G}_{\lambda}$ be two GIVFSM's. Then $\mathrm{F}_{\mu}$ is called GIVFS sub matrix of $\mathrm{G}_{\lambda}$ if

$F\left(e_{j}\right)\left(x_{i}\right) \leq G\left(e_{k}\right)\left(x_{i}\right)$ and $\mu\left(e_{j}\right) \leq, \lambda\left(e_{k}\right), \forall i=$ $1,2, \ldots, m$ and $j, k=1,2, \ldots, n$

Example 3.3. Consider a set of three motorbikes $U=$ $\left\{b_{1}, b_{2}, b_{3}\right\}$ and a set of parameters,

$E=\left\{e_{1}, e_{2}, e_{3}\right\}$, where $e_{1}, e_{2}$ and $e_{3}$ stands for cheap, expansive and comfortable respectively. The GFSS's $\mathrm{F}_{\mu}$ and $\mathrm{G}_{\lambda}$ are defined as

$$
\begin{aligned}
& \mathrm{F}_{\mu}\left(e_{1}\right) \\
& =\left\{\left(\frac{b_{1}}{[0.1,0.4]}, 0.3\right),\left(\frac{b_{2}}{[0.4,0.7]}, 0.3\right),\left(\frac{b_{3}}{[0.2,0.4]}, 0.3\right)\right\}
\end{aligned}
$$$$
\mathrm{F}_{\mu}\left(e_{2}\right)=\left\{\left(\frac{b_{1}}{[0.2,0.3]}, 0.4\right),\left(\frac{b_{2}}{[0,0.3]}, 0.4\right),\left(\frac{b_{3}}{[0.2,0.5]}, 0.4\right)\right\}
$$$$
\mathrm{F}_{\mu}\left(e_{3}\right)=\left\{\left(\frac{b_{1}}{[0.3,0.5]}, 0.1\right),\left(\frac{b_{2}}{[0.1,0.2]}, 0.1\right),\left(\frac{b_{3}}{[0,0.2]}, 0.1\right)\right\}
$$

and

$$
\begin{aligned}
& \mathrm{G}_{\lambda}\left(e_{1}\right) \\
& =\left\{\left(\frac{b_{1}}{[0.3,0.5]}, 0.4\right),\left(\frac{b_{2}}{[0.5,0.8]}, 0.4\right),\left(\frac{b_{3}}{[0.3,0.6]}, 0.4\right)\right\} \\
& \mathrm{G}_{\lambda}\left(e_{2}\right) \\
& =\left\{\left(\frac{b_{1}}{[0.4,0.5]}, 0.5\right),\left(\frac{b_{2}}{[0.2,0.4]}, 0.5\right),\left(\frac{b_{3}}{[0.4,0.6]}, 0.5\right)\right\} \\
& \mathrm{G}_{\lambda}\left(e_{3}\right) \\
& =\left\{\left(\frac{b_{1}}{[0.5,0.7]}, 0.3\right),\left(\frac{b_{2}}{[0.3,0.5]}, 0.3\right),\left(\frac{b_{3}}{[0.1,0.3]}, 0.3\right)\right\}
\end{aligned}
$$

Then the matrix representation of $\mathrm{F}_{\mu}$ and $\mathrm{G}_{\lambda}$ are given as

$$
\mathrm{F}_{\mu}=\left[\begin{array}{ccc}
([0.1,0.4], 0.3) & ([0.4,0.7], 0.3) & ([0.2,0.4], 0.3) \\
([0.2,0.3], 0.4) & ([0,0.3], 0.4) & ([0.2,0.5], 0.4) \\
([0.3,0.5], 0.1) & ([0.1,0.2], 0.1) & ([0,0.2], 0.1)
\end{array}\right]
$$

and

$$
\mathrm{G}_{\lambda}=\left[\begin{array}{lll}
([0.3,0.5], 0.4) & ([0.5,0.8], 0.4) & ([0.3,0.6], 0.4) \\
([0.4,0.5], 0.5) & ([0.2,0.4], 0.5) & ([0.4,0.6], 0.5) \\
([0.5,0.7], 0.3) & ([0.3,0.5], 0.3) & ([0.1,0.3], 0.3)
\end{array}\right]
$$

It is clear that $\mathrm{F}_{\mu}$ is GIVFS sub matrix of $\mathrm{G}_{\lambda}$.

Definition 3.4. Let $F_{\mu}$ and $G_{\lambda}$ be two GIVFSM's. Then $F_{\mu}$ is called GIVFS equal matrix of $\mathrm{G}_{\lambda}$ if

$F\left(e_{j}\right)\left(x_{i}\right)=G\left(e_{k}\right)\left(x_{i}\right)$ and $\mu\left(e_{j}\right)=\lambda\left(e_{k}\right), \forall i=1,2, \ldots, m$ and $j, k=1,2, \ldots, n$.

Definition 3.5. Let $\mathrm{F}_{\mu}$ and $\mathrm{G}_{\lambda}$ be two GIVFSM's. Then $\mathrm{F}_{\mu}$ is called GIVFS sub matrix of $\mathrm{G}_{\lambda}$ if

$F\left(e_{j}\right)\left(x_{i}\right) \leq G\left(e_{k}\right)\left(x_{i}\right), \mu\left(e_{j}\right) \leq \lambda\left(e_{k}\right), \forall e, F\left(e_{j}\right)\left(x_{i}\right)<$ $G\left(e_{k}\right)\left(x_{i}\right)$

and $\mu\left(e_{j}\right)<\lambda\left(e_{k}\right)$, for at least one $e$.

Definition 3.6. Let $\mathrm{F}_{\mu}$ and $\mathrm{G}_{\lambda}$ be two GIVFSM's. Then $\mathrm{F}_{\mu}$ is called GIVFS sub matrix of $\mathrm{G}_{\lambda}$ if

$F\left(e_{j}\right)\left(x_{i}\right)<G\left(e_{k}\right)\left(x_{i}\right)$ and $\mu\left(e_{j}\right)<\lambda\left(e_{k}\right), \forall i=1,2, \ldots, m$ and $j, k=1,2, \ldots, n$.

Definition 3.7. A GIVFSM $F_{\mu}$ is called GIVFS rectangular matrix if

$\mathrm{F}_{\mu}=\left[F\left(e_{j}\right)\left(x_{i}\right), \mu\left(e_{j}\right)\right]_{\mathrm{m} \times \mathrm{n}}$ and $\mathrm{m} \neq \mathrm{n}, \forall \mathrm{i}, \mathrm{j}$.

Definition 3.8. A GIVFSM $F_{\mu}$ is called GIVFS square matrix if

$\mathrm{F}_{\mu}=\left[F\left(e_{j}\right)\left(x_{i}\right), \mu\left(e_{j}\right)\right]_{\mathrm{m} \times \mathrm{n}}$ and $\mathrm{m}=\mathrm{n}, \forall \mathrm{i}, \mathrm{j}$.

Definition 3.9. A GIVFSM $F_{\mu}$ is called GIVFS diagonal matrix if

$\mathrm{F}_{\mu}=\left[F\left(e_{j}\right)\left(x_{i}\right), \mu\left(e_{j}\right) \quad\right]_{\mathrm{m} \times \mathrm{n}}, \quad \mathrm{m}=\mathrm{n} \quad$ and $\left(F\left(e_{j}\right)\left(x_{i}\right), \mu\left(e_{j}\right)\right)=(0,0), \forall \mathrm{i} \neq \mathrm{j}$.

Definition 3.10. A GIVFSM $\mathrm{F}_{\mu}$ is called GIVFS scalar matrix if

$\mathrm{F}_{\mu}=\left[F\left(e_{j}\right)\left(x_{i}\right), \mu\left(e_{j}\right)\right]_{\mathrm{m} \times \mathrm{n}}$ and $\left(F\left(e_{j}\right)\left(x_{i}\right), \mu\left(e_{j}\right)\right)=$ $(k, \mu), \forall \mathrm{i}=\mathrm{j}$.

Definition 3.11. A GIVFSM $F_{\mu}$ is called GIVFS row matrix if

$\mathrm{F}_{\mu}=\left[F\left(e_{j}\right)\left(x_{i}\right), \mu\left(e_{j}\right)\right]_{\mathrm{m} \times \mathrm{n}}$ and $\mathrm{m}=1, \forall \mathrm{i}, \mathrm{j}$.

Definition 3.12. A GIVFSM $F_{\mu}$ is called GIVFS column matrix if

$\mathrm{F}_{\mu}=\left[F\left(e_{j}\right)\left(x_{i}\right), \mu\left(e_{j}\right)\right]_{\mathrm{m} \times \mathrm{n}}$ and $\mathrm{n}=1, \forall \mathrm{i}, \mathrm{j}$.

Definition 3.13. Let $\mathrm{F}_{\mu}$ be a GIVFSM, then scalar multiple of $\mathrm{F}_{\mu}$ by a scalar $\mathrm{k}$ is defined as

$\mathrm{kF}_{\mu}=\left[k F\left(e_{j}\right)\left(x_{i}\right), k \mu\left(e_{j}\right)\right]_{\mathrm{m} \times \mathrm{n}}, \forall \mathrm{i}, \mathrm{j}$ and $0 \leq \mathrm{k} \leq 1$.

Definition 3.14. A GIVFSM $A_{\mu}$ is called generalized absolute IVFSM, if

$\mathrm{A}_{\mu}=\left[A\left(e_{j}\right)\left(x_{i}\right), \mu\left(e_{j}\right)\right]_{\mathrm{m} \times \mathrm{n}}$, where $A\left(e_{j}\right)\left(x_{i}\right)=$ 1 and $\mu\left(e_{j}\right)=1, \forall \mathrm{i}, \mathrm{j}$.

Definition 3.15. A GIVFSM $\phi_{\mu}$ is called generalized null IVFSM, if

$\phi_{\mu}=\left[\phi\left(e_{j}\right)\left(x_{i}\right), \mu\left(e_{j}\right)\right]_{\mathrm{m} \times \mathrm{n}}, \quad$ where $\phi\left(e_{j}\right)\left(x_{i}\right)=$ 0 and $\mu\left(e_{j}\right)=0, \forall \mathrm{i}, \mathrm{j}$.

\section{Some Operations on GIVFSM's}

Definition 4.1. Let $\mathrm{F}_{\mu}=\left[F\left(e_{j}\right)\left(x_{i}\right), \mu\left(e_{j}\right)\right]_{\mathrm{m} \times \mathrm{n}}$ be a GIVFSM, where

$F\left(e_{j}\right)\left(x_{i}\right)=\left[F\left(e_{j L}\right)\left(x_{i}\right), F\left(e_{j U}\right)\left(x_{i}\right)\right]$, then compliment of $\mathrm{F}_{\mu}$ is denoted by $\mathrm{F}_{\mu}{ }^{c}=\mathrm{G}_{\lambda}$ and is given by

$$
\mathrm{F}_{\mu}{ }^{c}=\mathrm{G}_{\lambda}=\left(\left[1-F\left(e_{j U}\right)\left(x_{i}\right), 1-F\left(e_{j L}\right)\left(x_{i}\right)\right], 1-\mu\left(e_{j}\right)\right), \forall \mathrm{i}, \mathrm{j} .
$$


Example 4.2. Consider a GIVFSM $\mathrm{F}_{\mu}$ as in example 3.3,

$\mathrm{F}_{\mu}=\left[\begin{array}{ccc}([0.1,0.4], 0.3) & ([0.4,0.7], 0.3) & ([0.2,0.4], 0.3) \\ ([0.2,0.3], 0.4) & ([0,0.3], 0.4) & ([0.2,0.5], 0.4) \\ ([0.3,0.5], 0.1) & ([0.1,0.2], 0.1) & ([0,0.2], 0.1)\end{array}\right]$

Then compliment of $\mathrm{F}_{\mu}$ is given by

$\mathrm{F}_{\mu}{ }^{c}=\left[\begin{array}{ccc}([0.6,0.9], 0.7) & ([0.3,0.6], 0.7) & ([0.6,0.8], 0.7) \\ ([0.7,0.8], 0.6) & ([0.7,1], 0.6) & ([0.5,0.8], 0.6) \\ ([0.5,0.7], 0.9) & ([0.8,0.9], 0.9) & ([0.8,1], 0.1)\end{array}\right]$

Proposition 4.3. Let $F_{\mu}$ be a GIVFSM, then $\left(F_{\mu}{ }^{c}\right)^{c}=F_{\mu}$

Proof:

Since

$$
\mathrm{F}_{\mu}{ }^{c}=\mathrm{G}_{\lambda}=\left(\left[F\left(e_{j U}\right)\left(x_{i}\right), F\left(e_{j L}\right)\left(x_{i}\right)\right], 1-\mu\left(e_{j}\right)\right)
$$

then $\left(\mathrm{F}_{\mu}{ }^{\mathrm{c}}\right)^{c}=\mathrm{G}_{\lambda}{ }^{c}$

but from definition, $\mathrm{G}_{\lambda}=\left(\left[F\left(e_{j U}\right)\left(x_{i}\right), F\left(e_{j L}\right)\left(x_{i}\right)\right], 1-\right.$ $\left.\mu\left(e_{j}\right)\right)$, then

$$
\begin{gathered}
\mathrm{G}_{\lambda}{ }^{c}=\left(\left[F\left(e_{j L}\right)\left(x_{i}\right), F\left(e_{j U}\right)\left(x_{i}\right)\right],\left(1-\left(1-\mu\left(e_{j}\right)\right)\right)\right)= \\
\left(\left[F\left(e_{j L}\right)\left(x_{i}\right), F\left(e_{j U}\right)\left(x_{i}\right)\right], \mu\left(e_{j}\right)\right)=\left[F\left(e_{j}\right)\left(x_{i}\right), \mu\left(e_{j}\right)\right]_{\mathrm{m} \times \mathrm{n}}= \\
\mathrm{F}_{\mu}
\end{gathered}
$$

Definition 4.4. The union of two GIVFSM's $\mathrm{F}_{\mu}=$ $\left[F\left(e_{j}\right)\left(x_{i}\right), \mu\left(e_{j}\right)\right]_{\mathrm{m} \times \mathrm{n}}$ and

$\mathrm{G}_{\lambda}=\left[G\left(e_{k}\right)\left(x_{i}\right), \lambda\left(e_{k}\right)\right]_{\mathrm{m} \times \mathrm{n}}$, denoted by $\mathrm{F}_{\mu} \cup \mathrm{G}_{\lambda}$ is a GIVFSM

$\left[H\left(e_{\ell}\right)\left(x_{i}\right), \gamma\left(e_{\ell}\right)\right]_{\mathrm{m} \times \mathrm{n}}$, such that

$\left[H\left(e_{\ell}\right)\left(x_{i}\right), \gamma\left(e_{\ell}\right)\right]_{\mathrm{m} \times \mathrm{n}}$

$=\left[\sup \left(F\left(e_{j L}\right), G\left(e_{k L}\right)\right), \sup \left(F\left(e_{j U}\right), G\left(e_{k U}\right)\right)\right]_{\mathrm{m} \times \mathrm{n}}$

$\gamma\left(e_{\ell}\right)=s\left(\mu\left(e_{j}\right), \lambda\left(e_{k}\right)\right)$, where $s$ is an $s-$ norm.

Example 4.5. Consider two GIVFSM's $\mathrm{F}_{\mu}$ and $\mathrm{G}_{\lambda}$ as in example 3.3,

$$
\mathrm{F}_{\mu}=\left[\begin{array}{ccc}
([0.1,0.4], 0.3) & ([0.4,0.7], 0.3) & ([0.2,0.4], 0.3) \\
([0.2,0.3], 0.4) & ([0,0.3], 0.4) & ([0.2,0.5], 0.4) \\
([0.3,0.5], 0.1) & ([0.1,0.2], 0.1) & ([0,0.2], 0.1)
\end{array}\right]
$$

and

$$
\mathrm{G}_{\lambda}=\left[\begin{array}{lll}
([0.3,0.5], 0.4) & ([0.5,0.8], 0.4) & ([0.3,0.6], 0.4) \\
([0.4,0.5], 0.5) & ([0.2,0.4], 0.5) & ([0.4,0.6], 0.5) \\
([0.5,0.7], 0.3) & ([0.3,0.5], 0.3) & ([0.1,0.3], 0.3)
\end{array}\right]
$$

Then the union of $F_{\mu}$ and $G_{\lambda}$ is given by

$$
\begin{aligned}
& \mathrm{F}_{\mu} \cup \mathrm{G}_{\lambda} \\
& =\left[\begin{array}{lll}
([0.3,0.5], 0.4) & ([0.5,0.8], 0.4) & ([0.3,0.6], 0.4) \\
([0.4,0.5], 0.5) & ([0.2,0.4], 0.5) & ([0.4,0.6], 0.5) \\
([0.5,0.7], 0.3) & ([0.3,0.5], 0.3) & ([0.1,0.3], 0.3)
\end{array}\right]
\end{aligned}
$$

Proposition 4.6. Let $F_{\mu}$ be a GIVFSM, then

$$
F_{\mu} \cup F_{\mu}=F_{\mu}
$$

Proof: From Definition, we have

$$
\begin{gathered}
{\left[F\left(e_{j}\right)\left(x_{i}\right), \mu\left(e_{j}\right)\right]_{\mathrm{m} \times \mathrm{n}} \cup\left[F\left(e_{j}\right)\left(x_{i}\right), \mu\left(e_{j}\right)\right]_{\mathrm{m} \times \mathrm{n}}} \\
=\left[H\left(e_{j}\right)\left(x_{i}\right), \gamma\left(e_{j}\right)\right]_{\mathrm{m} \times \mathrm{n}}
\end{gathered}
$$

Such that

$$
\begin{gathered}
{\left[H\left(e_{j}\right)\left(x_{i}\right), \gamma\left(e_{j}\right)\right]_{\mathrm{m} \times \mathrm{n}}} \\
=\left[\sup \left(F\left(e_{j L}\right), F\left(e_{j L}\right)\right), \sup \left(F\left(e_{j U}\right), F\left(e_{j U}\right)\right)\right]_{\mathrm{m} \times \mathrm{n}} \\
=\left[F\left(e_{j}\right)\left(x_{i}\right), \mu\left(e_{j}\right)\right]_{\mathrm{m} \times \mathrm{n}} \\
=\mathrm{F}_{\mu}
\end{gathered}
$$

Proposition 4.7. Let $F_{\mu}=\left[F\left(e_{j}\right)\left(x_{i}\right), \mu\left(e_{j}\right)\right]_{\mathrm{m} \times \mathrm{n}}$ and $G_{\lambda}=$ $\left[G\left(e_{k}\right)\left(x_{i}\right), \lambda\left(e_{k}\right)\right]_{\mathrm{m} \times \mathrm{n}}$ be two GIVFSM's, then

a) $F_{\mu} \cup G_{\lambda}=G_{\lambda} \cup F_{\mu}$

b) $F_{\mu} \cup A_{\mu}=A_{\mu}$

c) $F_{\mu} \cup \phi_{\mu}=F_{\mu}$

Proof:

(a) From Definition, we have

$$
\begin{gathered}
{\left[F\left(e_{j}\right)\left(x_{i}\right), \mu\left(e_{j}\right)\right]_{\mathrm{m} \times \mathrm{n}} \cup\left[G\left(e_{k}\right)\left(x_{i}\right), \lambda\left(e_{k}\right)\right]_{\mathrm{m} \times \mathrm{n}}} \\
=\left[H\left(e_{\ell}\right)\left(x_{i}\right), \gamma\left(e_{\ell}\right)\right]_{\mathrm{m} \times \mathrm{n}}
\end{gathered}
$$

Such that

$$
\begin{aligned}
& {\left[H\left(e_{\ell}\right)\left(x_{i}\right), \gamma\left(e_{\ell}\right)\right]_{\mathrm{m} \times \mathrm{n}}} \\
& =\left[\sup \left(F\left(e_{j L}\right), G\left(e_{k L}\right)\right), \sup \left(F\left(e_{j U}\right), G\left(e_{k U}\right)\right)\right]_{\mathrm{m} \times \mathrm{n}}
\end{aligned}
$$

But $\mathrm{H}_{\gamma}=\mathrm{F}_{\mu} \cup \mathrm{G}_{\lambda}=\mathrm{G}_{\lambda} \cup \mathrm{F}_{\mu}$ (since union of GIVFSM's is commutative)

and $\quad \gamma\left(e_{\ell}\right)=s\left(\mu\left(e_{j}\right), \lambda\left(e_{k}\right)\right)=s\left(\lambda\left(e_{k}\right), \mu\left(e_{j}\right)\right), \quad$ (since $s-$ norm is commutative)

Then,

$$
\mathrm{F}_{\mu} \cup \mathrm{G}_{\lambda}=\mathrm{G}_{\lambda} \cup \mathrm{F}_{\mu}
$$

The proof of (b) and (c) are straight forward from definition.

Definition 4.8. The intersection of two GIVFSM's $\mathrm{F}_{\mu}=$ $\left[F\left(e_{j}\right)\left(x_{i}\right), \mu\left(e_{j}\right)\right]_{\mathrm{m} \times \mathrm{n}}$ and

$\mathrm{G}_{\lambda}=\left[G\left(e_{k}\right)\left(x_{i}\right), \lambda\left(e_{k}\right)\right]_{\mathrm{m} \times \mathrm{n}}$, denoted by $\mathrm{F}_{\mu} \cap \mathrm{G}_{\lambda}$ is a $\operatorname{GIVFSM}\left[H\left(e_{\ell}\right)\left(x_{i}\right), \gamma\left(e_{\ell}\right)\right]_{\mathrm{m} \times \mathrm{n}}$, such that

$$
\begin{aligned}
& {\left[H\left(e_{\ell}\right)\left(x_{i}\right), \gamma\left(e_{\ell}\right)\right]_{\mathrm{m} \times \mathrm{n}}} \\
& =\left[\inf \left(F\left(e_{j L}\right), G\left(e_{k L}\right)\right), \inf \left(F\left(e_{j U}\right), G\left(e_{k U}\right)\right)\right]_{\mathrm{m} \times \mathrm{n}}
\end{aligned}
$$

$\gamma\left(e_{\ell}\right)=t\left(\mu\left(e_{j}\right), \lambda\left(e_{k}\right)\right)$, where $t$ is a $t-$ norm.

Example 4.9. Consider two GIVFSM's $\mathrm{F}_{\mu}$ and $\mathrm{G}_{\lambda}$ as in example 3.3,

$$
\mathrm{F}_{\mu}=\left[\begin{array}{ccc}
([0.1,0.4], 0.3) & ([0.4,0.7], 0.3) & ([0.2,0.4], 0.3) \\
([0.2,0.3], 0.4) & ([0,0.3], 0.4) & ([0.2,0.5], 0.4) \\
([0.3,0.5], 0.1) & ([0.1,0.2], 0.1) & ([0,0.2], 0.1)
\end{array}\right]
$$


and

$$
\mathrm{G}_{\lambda}=\left[\begin{array}{lll}
([0.3,0.5], 0.4) & ([0.5,0.8], 0.4) & ([0.3,0.6], 0.4) \\
([0.4,0.5], 0.5) & ([0.2,0.4], 0.5) & ([0.4,0.6], 0.5) \\
([0.5,0.7], 0.3) & ([0.3,0.5], 0.3) & ([0.1,0.3], 0.3)
\end{array}\right]
$$

Then the intersection of $F_{\mu}$ and $G_{\lambda}$ is given by

$$
\begin{aligned}
& \mathrm{F}_{\mu} \cap \mathrm{G}_{\lambda} \\
& =\left[\begin{array}{ccc}
([0.1,0.4], 0.3) & ([0.4,0.7], 0.3) & ([0.2,0.4], 0.3) \\
([0.2,0.3], 0.4) & ([0,0.3], 0.4) & ([0.2,0.5], 0.4) \\
([0.3,0.5], 0.1) & ([0.1,0.2], 0.1) & ([0,0.2], 0.1)
\end{array}\right]
\end{aligned}
$$

Proposition 4.10. Let $F_{\mu}$ be a GIVFSM, then

$$
F_{\mu} \cap F_{\mu}=F_{\mu}
$$

Proof: From Definition, we have

$$
\begin{gathered}
{\left[F\left(e_{j}\right)\left(x_{i}\right), \mu\left(e_{j}\right)\right]_{\mathrm{m} \times \mathrm{n}} \cap\left[F\left(e_{j}\right)\left(x_{i}\right), \mu\left(e_{j}\right)\right]_{\mathrm{m} \times \mathrm{n}}} \\
=\left[H\left(e_{\ell}\right)\left(x_{i}\right), \gamma\left(e_{\ell}\right)\right]_{\mathrm{m} \times \mathrm{n}}
\end{gathered}
$$

such that

$$
\begin{gathered}
{\left[H\left(e_{\ell}\right)\left(x_{i}\right), \gamma\left(e_{\ell}\right)\right]_{\mathrm{m} \times \mathrm{n}}} \\
=\left[\inf \left(F\left(e_{j L}\right), F\left(e_{j L}\right)\right), \inf \left(F\left(e_{j U}\right), F\left(e_{j U}\right)\right)\right]_{\mathrm{m} \times \mathrm{n}} \\
=\left[F\left(e_{j}\right)\left(x_{i}\right), \mu\left(e_{j}\right)\right]_{\mathrm{m} \times \mathrm{n}} \\
=\mathrm{F}_{\mu}
\end{gathered}
$$

Proposition 4.11. Let $F_{\mu}=\left[F\left(e_{j}\right)\left(x_{i}\right), \mu\left(e_{j}\right)\right]_{\mathrm{m} \times \mathrm{n}}$ and $G_{\lambda}=$ $\left[G\left(e_{k}\right)\left(x_{i}\right), \lambda\left(e_{k}\right)\right]_{\mathrm{m} \times \mathrm{n}}$ be two GIVFSM's, then

(a) $F_{\mu} \cap G_{\lambda}=G_{\lambda} \cap F_{\mu}$

Proof:

(a) Consider $\mathrm{F}_{\mu}{ }^{c} \cap \mathrm{G}_{\lambda}{ }^{c}=\left(\left[F\left(e_{j U}\right)\left(x_{i}\right), F\left(e_{j L}\right)\left(x_{i}\right)\right], 1-\mu\left(e_{j}\right)\right) \cap\left(\left[G\left(e_{k U}\right)\left(x_{i}\right), G\left(e_{k L}\right)\left(x_{i}\right)\right], 1-\lambda\left(e_{k}\right)\right)$

$$
\begin{gathered}
=\left(\left[\inf \left(F\left(e_{j L}\right), G\left(e_{k L}\right)\right), \inf \left(F\left(e_{j U}\right), G\left(e_{k U}\right)\right)\right],\left(1-\mu\left(e_{j}\right)\right) \cap\left(1-\lambda\left(e_{k}\right)\right)\right) \\
=\left(\left(\left[F\left(e_{j U}\right)\left(x_{i}\right), F\left(e_{j L}\right)\left(x_{i}\right)\right] \cup\left[G\left(e_{k U}\right)\left(x_{i}\right), G\left(e_{k L}\right)\left(x_{i}\right)\right]\right)^{c},\left(\left(1-\mu\left(e_{j}\right)\right) \cup\left(1-\lambda\left(e_{k}\right)\right)\right)^{c}\right) \\
=\left(\left[F\left(e_{j}\right)\left(x_{i}\right), \mu\left(e_{j}\right)\right] \cup\left[G\left(e_{k}\right)\left(x_{i}\right), \lambda\left(e_{k}\right)\right]\right)^{c} \\
=\left(\mathrm{F}_{\mu} \cup \mathrm{G}_{\lambda}\right)^{c}
\end{gathered}
$$

(b) The proof is similar to proof of (a).

Proposition 4.14. Let $F_{\mu}=\left[F\left(e_{j}\right)\left(x_{i}\right), \mu\left(e_{j}\right)\right]_{\mathrm{m} \times \mathrm{n}}, G_{\lambda}=\left[G\left(e_{k}\right)\left(x_{i}\right), \lambda\left(e_{k}\right)\right]_{\mathrm{m} \times \mathrm{n}}$ and $H_{\gamma}=\left[H\left(e_{\ell}\right)\left(x_{i}\right), \gamma\left(e_{\ell}\right)\right]_{\mathrm{m} \times \mathrm{n}}$ be three GIVFSM's, then

(a) $F_{\mu} \cup\left(G_{\lambda} \cap H_{\gamma}\right)=\left(F_{\mu} \cup G_{\lambda}\right) \cap\left(F_{\mu} \cup H_{\gamma}\right)$

(b) $F_{\mu} \cap\left(G_{\lambda} \cup H_{\gamma}\right)=\left(F_{\mu} \cap G_{\lambda}\right) \cup\left(F_{\mu} \cap H_{\gamma}\right)$

Proof:

(a) $\mathrm{F}_{\mu} \cup\left(\mathrm{G}_{\lambda} \cap \mathrm{H}_{\gamma}\right)=\left[\sup \left(F\left(e_{j L}\right),\left(\mathrm{G}\left(\mathrm{e}_{\mathrm{kL}}\right) \cap \mathrm{H}\left(\mathrm{e}_{\ell \mathrm{L}}\right)\right), \sup \left(F\left(e_{j U}\right),\left(\mathrm{G}\left(\mathrm{e}_{\mathrm{kU}}\right) \cap H\left(e_{\ell U}\right)\right)\right]\right.\right.$

$$
\begin{gathered}
=\left[\sup \left(F\left(e_{j L}\right), \inf \left(G\left(e_{j L}\right), H\left(e_{\ell L}\right)\right)\right), \sup \left(F\left(e_{j U}\right), \inf \left(G\left(e_{j U}\right), H\left(e_{\ell U}\right)\right)\right)\right] \\
=\left[\operatorname { i n f } \left(\operatorname { s u p } \left(F\left(e_{j L}\right),\left(\mathrm{G}\left(\mathrm{e}_{\mathrm{kL}}\right)\right), \sup \left(\left(F\left(e_{j L}\right), H\left(e_{\ell L}\right)\right)\right), \inf \left(\sup \left(F\left(e_{j U}\right),\left(G\left(e_{j U}\right)\right), \sup \left(\left(F\left(e_{j U}\right), H\left(e_{\ell U}\right)\right)\right)\right]\right.\right.\right.\right.
\end{gathered}
$$




$$
=\left(F\left(e_{j L}\right), F\left(e_{j U}\right)\right) \cup\left(G\left(e_{k L}\right), G\left(e_{k U}\right)\right) \cap\left(\left(F\left(e_{j L}\right), F\left(e_{j U}\right)\right) \cup\left(H\left(e_{\ell L}\right), H\left(e_{\ell U}\right)\right)\right)
$$

and

$$
\begin{gathered}
\left(\mu\left(e_{j}\right) \cup\left(\lambda\left(e_{k}\right) \cap \gamma\left(e_{\ell}\right)\right)=\max \left\{\left(\mu\left(e_{j}\right),\left(\lambda\left(e_{k}\right) \cap \gamma\left(e_{\ell}\right)\right\}\right.\right.\right. \\
=\max \left\{\mu\left(e_{j}\right), \min \left(\lambda\left(e_{k}\right) \cap \gamma\left(e_{\ell}\right)\right)\right\} \\
=\min \left\{\max \left(\mu\left(e_{j}\right), \lambda\left(e_{k}\right)\right), \max \left(\mu\left(e_{j}\right), \gamma\left(e_{\ell}\right)\right)\right\} \\
=\min \left\{\mu\left(e_{j}\right) \cup \lambda\left(e_{k}\right),\left(\mu\left(e_{j}\right) \cup\left(\gamma\left(e_{\ell}\right)\right\}\right.\right. \\
=\left(\mu\left(e_{j}\right) \cup \lambda\left(e_{k}\right)\right) \cap\left(\left(\mu\left(e_{j}\right) \cup\left(\gamma\left(e_{\ell}\right)\right)\right.\right.
\end{gathered}
$$

(b) The proof is similar to proof of (a).

Definition 4.15. Let $\mathrm{F}_{\mu}=\left[F\left(e_{j}\right)\left(x_{i}\right), \mu\left(e_{j}\right)\right]_{\mathrm{m} \times \mathrm{n}}$ and $\mathrm{G}_{\lambda}=$ $\left[G\left(e_{k}\right)\left(x_{i}\right), \lambda\left(e_{k}\right)\right]_{\mathrm{m} \times \mathrm{n}}$ be two GIVFSM's, then AND product of $F_{\mu}$ and $G_{\lambda}$ is denoted by $F_{\mu} \wedge G_{\lambda}$ and is defined as

$$
\mathrm{F}_{\mu} \wedge \mathrm{G}_{\lambda}=\mathrm{H}_{\gamma}
$$

Such that $\mathrm{H}_{\gamma}=\left[H\left(e_{\ell}\right)\left(x_{i}\right), \gamma\left(e_{\ell}\right)\right]_{\mathrm{m} \times \mathrm{n}}$

where $H\left(e_{\ell}\right)\left(x_{i}\right)=F\left(e_{j}\right)\left(x_{i}\right) \cap G\left(e_{k}\right)\left(x_{i}\right), F\left(e_{j}\right), G\left(e_{k}\right) \in$ $F\left(e_{j}\right) \times G\left(e_{k}\right) \forall x_{i}, e_{j}, e_{k}$.

and $\gamma\left(e_{\ell}\right)=t\left(\mu\left(e_{j}\right), \lambda\left(e_{k}\right)\right)$, where $\left.\mu\left(e_{j}\right), \lambda\left(e_{k}\right)\right), \in \mu\left(e_{j}\right) \times$ $\lambda\left(e_{k}\right) \forall \mathrm{j}, \mathrm{k}$ and $t$ is a $t-$ norm.

Example 4.16. Consider two GIVFSM's $\mathrm{F}_{\mu}$ and $\mathrm{G}_{\lambda}$ as in example 3.3,

$$
\mathrm{F}_{\mu}=\left[\begin{array}{ccc}
([0.1,0.4], 0.3) & ([0.4,0.7], 0.3) & ([0.2,0.4], 0.3) \\
([0.2,0.3], 0.4) & ([0,0.3], 0.4) & ([0.2,0.5], 0.4) \\
([0.3,0.5], 0.1) & ([0.1,0.2], 0.1) & ([0,0.2], 0.1)
\end{array}\right]
$$

and

$$
G_{\lambda}=\left[\begin{array}{lll}
([0.3,0.5], 0.4) & ([0.5,0.8], 0.4) & ([0.3,0.6], 0.4) \\
([0.4,0.5], 0.5) & ([0.2,0.4], 0.5) & ([0.4,0.6], 0.5) \\
([0.5,0.7], 0.3) & ([0.3,0.5], 0.3) & ([0.1,0.3], 0.3)
\end{array}\right]
$$

Then $F_{\mu} \wedge G_{\lambda}$ is given by

$$
\begin{aligned}
& \mathrm{F}_{\mu} \wedge \mathrm{G}_{\lambda} \\
& =\left[\begin{array}{ccc}
([0.1,0.4], 0.3) & ([0.4,0.7], 0.3) & ([0.2,0.4], 0.3) \\
([0.1,0.4], 0.3) & ([0.2,0.4], 0.3) & ([0.2,0.4], 0.3) \\
([0.1,0.4], 0.3) & ([0.3,0.5], 0.3) & ([0.1,0.3], 0.3) \\
([0.2,0.3], 0.4) & ([0,0.3], 0.4) & ([0.2,0.5], 0.4) \\
([0.2,0.3], 0.4) & ([0,0.3], 0.4) & ([0.2,0.5], 0.4) \\
([0.2,0.3], 0.3) & ([0,0.3], 0.3) & ([0.1,0.3], 0.3) \\
([0.3,0.5], 0.1) & ([0.1,0.2], 0.1) & ([0,0.2], 0.1) \\
([0.3,0.5], 0.1) & ([0.1,0.2], 0.1) & ([0,0.2], 0.1) \\
([0.3,0.5], 0.1) & ([0.1,0.2], 0.1) & ([0,0.2], 0.1)
\end{array}\right]
\end{aligned}
$$

Definition 4.17. Let $\mathrm{F}_{\mu}=\left[F\left(e_{j}\right)\left(x_{i}\right), \mu\left(e_{j}\right)\right]_{\mathrm{m} \times \mathrm{n}}$ and $\mathrm{G}_{\lambda}=$ $\left[G\left(e_{k}\right)\left(x_{i}\right), \lambda\left(e_{k}\right)\right]_{\mathrm{m} \times \mathrm{n}}$ be two GIVFSM's, then OR product of $F_{\mu}$ and $G_{\lambda}$ is denoted by $F_{\mu} \vee G_{\lambda}$ and is defined as

$$
\mathrm{F}_{\mu} \vee \mathrm{G}_{\lambda}=\mathrm{H}_{\gamma}
$$

Such that $\mathrm{H}_{\gamma}=\left[H\left(e_{\ell}\right)\left(x_{i}\right), \gamma\left(e_{\ell}\right)\right]_{\mathrm{m} \times \mathrm{n}}$ where

$$
\begin{aligned}
H\left(e_{\ell}\right)\left(x_{i}\right)= & F\left(e_{j}\right)\left(x_{i}\right) \cap G\left(e_{j}\right)\left(x_{i}\right), F\left(e_{j}\right), G\left(e_{k}\right) \in \\
& F\left(e_{j}\right) \times G\left(e_{k}\right) \forall x_{i}, e_{j}, e_{k} .
\end{aligned}
$$

and

$$
\begin{gathered}
\left.\gamma\left(e_{\ell}\right)=s\left(\mu\left(e_{j}\right), \lambda\left(e_{k}\right)\right), \text { where } \mu\left(e_{j}\right), \lambda\left(e_{k}\right)\right), \in \mu\left(e_{j}\right) \times \\
\lambda\left(e_{k}\right) \forall \mathrm{j}, \mathrm{k} \text { and } s \text { is an } s-\text { norm. }
\end{gathered}
$$

Example 4.18. Consider two GIVFSM's $\mathrm{F}_{\mu}$ and $\mathrm{G}_{\lambda}$ as in example 3.3,

$$
\mathrm{F}_{\mu}=\left[\begin{array}{ccc}
([0.1,0.4], 0.3) & ([0.4,0.7], 0.3) & ([0.2,0.4], 0.3) \\
([0.2,0.3], 0.4) & ([0,0.3], 0.4) & ([0.2,0.5], 0.4) \\
([0.3,0.5], 0.1) & ([0.1,0.2], 0.1) & ([0,0.2], 0.1)
\end{array}\right]
$$

and

$$
G_{\lambda}=\left[\begin{array}{lll}
([0.3,0.5], 0.4) & ([0.5,0.8], 0.4) & ([0.3,0.6], 0.4) \\
([0.4,0.5], 0.5) & ([0.2,0.4], 0.5) & ([0.4,0.6], 0.5) \\
([0.5,0.7], 0.3) & ([0.3,0.5], 0.3) & ([0.1,0.3], 0.3)
\end{array}\right]
$$

Then $\mathrm{F}_{\mu} \vee \mathrm{G}_{\lambda}$ is given by

$$
\begin{aligned}
& \mathrm{F}_{\mu} \vee \mathrm{G}_{\lambda} \\
& \left.\quad \begin{array}{lcc}
([0.1,0.4], 0.4) & ([0.4,0.7], 0.4) & ([0.2,0.4], 0.4) \\
([0.1,0.4], 0.5) & ([0.2,0.4], 0.5) & ([0.2,0.4], 0.5) \\
([0.1,0.4], 0.3) & ([0.3,0.5], 0.3) & ([0.1,0.3], 0.3) \\
([0.2,0.3], 0.4) & ([0,0.3], 0.4) & ([0.2,0.5], 0.4) \\
([0.2,0.3], 0.5) & ([0,0.3], 0.5) & ([0.2,0.5], 0.5) \\
([0.2,0.3], 0.3) & ([0,0.3], 0.3) & ([0.1,0.3], 0.3) \\
([0.3,0.5], 0.4) & ([0.1,0.2], 0.4) & ([0,0.2], 0.4) \\
([0.3,0.5], 0.5) & ([0.1,0.2], 0.5) & ([0,0.2], 0.5) \\
([0.3,0.5], 0.3) & ([0.1,0.2], 0.3) & ([0,0.2], 0.3)
\end{array}\right]
\end{aligned}
$$

Definition 4.19. Let $\mathrm{F}_{\mu}=\left[F\left(e_{j}\right)\left(x_{i}\right), \mu\left(e_{j}\right)\right]_{\mathrm{m} \times \mathrm{n}}$ and $\mathrm{G}_{\lambda}=$ $\left[G\left(e_{k}\right)\left(x_{i}\right), \lambda\left(e_{k}\right)\right]_{\mathrm{m} \times \mathrm{n}}$ be two GIVFSM's, then arithmetic mean of $F_{\mu}$ and $G_{\lambda}$, denoted by $F_{\mu} @ G_{\lambda}$ is defined by

$$
\mathrm{F}_{\mu} @ \mathrm{G}_{\lambda}=\mathrm{H}_{\gamma},
$$

where

$$
\mathrm{H}_{\gamma}=\left[H\left(e_{\ell}\right)\left(x_{i}\right), \gamma\left(e_{\ell}\right)\right]
$$

such that

$$
\begin{gathered}
H\left(e_{\ell}\right)\left(x_{i}\right)=\left[H\left(e_{\ell L}\right)\left(x_{i}\right), H\left(e_{\ell U}\right)\left(x_{i}\right)\right] \\
H\left(e_{\ell L}\right)\left(x_{i}\right)=\frac{\frac{F\left(e_{j L}\right)\left(x_{i}\right)+G\left(e_{k L}\right)\left(x_{i}\right)}{2}, H\left(e_{\ell U}\right)\left(x_{i}\right)=}{\frac{F\left(e_{j U}\right)\left(x_{i}\right)+G\left(e_{k U}\right)\left(x_{i}\right)}{2}}
\end{gathered}
$$

and

$$
\gamma\left(e_{\ell}\right)=\frac{\mu\left(e_{j}\right)+\lambda\left(e_{k}\right)}{2}
$$


Definition 4.20. Let $\mathrm{F}_{\mu}=\left[F\left(e_{j}\right)\left(x_{i}\right), \mu\left(e_{j}\right)\right]_{\mathrm{m} \times \mathrm{n}}$ and $\mathrm{G}_{\lambda}=$ $\left[G\left(e_{k}\right)\left(x_{i}\right), \lambda\left(e_{k}\right)\right]_{\mathrm{m} \times \mathrm{n}}$ be two GIVFSM's, then weighted arithmetic mean of $\mathrm{F}_{\mu}$ and $\mathrm{G}_{\lambda}$, denoted by $\mathrm{F}_{\mu} @{ }^{w} \mathrm{G}_{\lambda}$ is defined by

$$
\mathrm{F}_{\mu} @^{w} \mathrm{G}_{\lambda}=\mathrm{H}_{\gamma}{ }^{w},
$$

Where

$$
\mathrm{H}_{\gamma}{ }^{w}=\left[H^{w}\left(e_{\ell}\right)\left(x_{i}\right), \gamma^{w}\left(e_{\ell}\right)\right]
$$

such that

Definition 4.21. Let $\mathrm{F}_{\mu}=\left[F\left(e_{j}\right)\left(x_{i}\right), \mu\left(e_{j}\right)\right]_{\mathrm{m} \times \mathrm{n}}$ and $\mathrm{G}_{\lambda}=\left[G\left(e_{k}\right)\left(x_{i}\right), \lambda\left(e_{k}\right)\right]_{\mathrm{m} \times \mathrm{n}}$ be two GIVFSM's, then geometric mean of $\mathrm{F}_{\mu}$ and $\mathrm{G}_{\lambda}$, denoted by $\mathrm{F}_{\mu} \$ \mathrm{G}_{\lambda}$ is defined by

$$
\mathrm{F}_{\mu} \$ \mathrm{G}_{\lambda}=\left(\left[\sqrt{F\left(e_{j L}\right)\left(x_{i}\right) \cdot G\left(e_{k L}\right)\left(x_{i}\right)}, \sqrt{F\left(e_{j U}\right)\left(x_{i}\right) \cdot G\left(e_{k U}\right)\left(x_{i}\right)}\right], \sqrt{\mu\left(e_{j}\right) \cdot \lambda\left(e_{k}\right)}\right)
$$

Definition 4.22. Let $\mathrm{F}_{\mu}=\left[F\left(e_{j}\right)\left(x_{i}\right), \mu\left(e_{j}\right)\right]_{\mathrm{m} \times \mathrm{n}}$ and $\mathrm{G}_{\lambda}=\left[G\left(e_{k}\right)\left(x_{i}\right), \lambda\left(e_{k}\right)\right]_{\mathrm{m} \times \mathrm{n}}$ be two GIVFSM's, then weighted geometric mean of $F_{\mu}$ and $G_{\lambda}$, denoted by $F_{\mu} \$^{w} G_{\lambda}$ is defined by

$$
\begin{gathered}
\mathrm{F}_{\mu} \$^{w} \mathrm{G}_{\lambda} \\
=\left(\left[\left(F\left(e_{j L}\right)\left(x_{i}\right)^{w_{1}} \cdot G\left(e_{k L}\right)\left(x_{i}\right)^{w_{2}}\right)^{\frac{1}{w_{1}+w_{2}}},\left(F\left(e_{j U}\right)\left(x_{i}\right)^{w_{1}} \cdot G\left(e_{k U}\right)\left(x_{i}\right)^{w_{2}}\right)^{\frac{1}{w_{1}+w_{2}}}\right],\left(\mu\left(e_{j}\right)^{w_{1}} \cdot \lambda\left(e_{k}\right)^{w_{2}}\right)^{\frac{1}{w_{1}+w_{2}}}\right)
\end{gathered}
$$

Definition 4.23. Let $\mathrm{F}_{\mu}=\left[F\left(e_{j}\right)\left(x_{i}\right), \mu\left(e_{j}\right)\right]_{\mathrm{m} \times \mathrm{n}}$ and $\mathrm{G}_{\lambda}=\left[G\left(e_{k}\right)\left(x_{i}\right), \lambda\left(e_{k}\right)\right]_{\mathrm{m} \times \mathrm{n}}$ be two GIVFSM's, then harmonic mean of $\mathrm{F}_{\mu}$ and $\mathrm{G}_{\lambda}$, denoted by $\mathrm{F}_{\mu} \& \mathrm{G}_{\lambda}$ is defined by

$$
\mathrm{F}_{\mu} \& \mathrm{G}_{\lambda}=\left(\left[2 \cdot \frac{F\left(e_{j L}\right)\left(x_{i}\right) \cdot G\left(e_{k L}\right)\left(x_{i}\right)}{F\left(e_{j L}\right)\left(x_{i}\right)+G\left(e_{k L}\right)\left(x_{i}\right)}, 2 \cdot \frac{F\left(e_{j U}\right)\left(x_{i}\right) \cdot G\left(e_{k U}\right)\left(x_{i}\right)}{F\left(e_{j U}\right)\left(x_{i}\right)+G\left(e_{k U}\right)\left(x_{i}\right)}\right], 2 \cdot \frac{\mu\left(e_{j}\right) \cdot \lambda\left(e_{k}\right)}{\mu\left(e_{j}\right)+\lambda\left(e_{k}\right)}\right)
$$

Definition 4.24. Let $\mathrm{F}_{\mu}=\left[F\left(e_{j}\right)\left(x_{i}\right), \mu\left(e_{j}\right)\right]_{\mathrm{m} \times \mathrm{n}}$ and $\mathrm{G}_{\lambda}=\left[G\left(e_{k}\right)\left(x_{i}\right), \lambda\left(e_{k}\right)\right]_{\mathrm{m} \times \mathrm{n}}$ be two GIVFSM's, then weighted harmonic mean of $\mathrm{F}_{\mu}$ and $\mathrm{G}_{\lambda}$, denoted by $\mathrm{F}_{\mu} \&^{w} \mathrm{G}_{\lambda}$ is defined by

$$
\mathrm{F}_{\mu} \&^{w} \mathrm{G}_{\lambda}=\left(\left[\frac{w_{1}+w_{2}}{\frac{w_{1}}{F\left(e_{j L}\right)\left(x_{i}\right)}+\frac{w_{2}}{G\left(e_{k L}\right)\left(x_{i}\right)}}, \frac{w_{1}+w_{2}}{\frac{w_{1}}{F\left(e_{j U}\right)\left(x_{i}\right)}+\frac{w_{2}}{G\left(e_{k U}\right)\left(x_{i}\right)}}\right], \frac{w_{1}+w_{2}}{\frac{w_{1}}{\mu\left(e_{j}\right)}+\frac{w_{2}}{\lambda\left(e_{k}\right)}}\right)
$$

Proposition 4.25. Let $F_{\mu}=\left[F\left(e_{j}\right)\left(x_{i}\right), \mu\left(e_{j}\right)\right]_{\mathrm{m} \times \mathrm{n}}$ and $G_{\lambda}=\left[G\left(e_{k}\right)\left(x_{i}\right), \lambda\left(e_{k}\right)\right]_{\mathrm{m} \times \mathrm{n}}$ be two GIVFSM's, then the following holds.

(a) $\quad F_{\mu} @ G_{\lambda}=G_{\lambda} @ F_{\mu}$

(b) $F_{\mu} \$ G_{\lambda}=\$ G_{\lambda} F_{\mu}$

(c) $F_{\mu} \& G_{\lambda}=G_{\lambda} \& F_{\mu}$

\section{Similarity Between Two GIVESM's}

Definition 5.1. Let $\mathrm{F}_{\mu}=\left[F\left(e_{j}\right)\left(x_{i}\right), \mu\left(e_{j}\right)\right]_{\mathrm{m} \times \mathrm{n}}$ and $\mathrm{G}_{\lambda}=$ [ $\left.G\left(e_{k}\right)\left(x_{i}\right), \lambda\left(e_{k}\right)\right]_{\mathrm{m} \times \mathrm{n}}$ be two GIVFSM's, then similarity between $\mathrm{F}_{\mu}$ and $\mathrm{G}_{\lambda}$, denoted by $S\left(\mathrm{~F}_{\mu}, \mathrm{G}_{\lambda}\right)$, is defined by

$$
\begin{aligned}
& S\left(\mathrm{~F}_{\mu}, \mathrm{G}_{\lambda}\right)=\mathrm{H}_{\gamma}, \text { where } \mathrm{H}_{\gamma}=\left[H\left(e_{\ell L}\right)\left(x_{i}\right) \cdot \gamma, H\left(e_{\ell U}\right)\left(x_{i}\right) \cdot \gamma\right], \\
& H\left(e_{\ell L}\right)\left(x_{i}\right) \\
&=\min \left(\varphi\left(F\left(e_{j L}\right), G\left(e_{k L}\right)\right), \phi\left(F\left(e_{j U}\right), G\left(e_{k U}\right)\right)\right) \\
& H\left(e_{\ell U}\right)\left(x_{i}\right) \\
&=\max \left(\varphi\left(F\left(e_{j L}\right), G\left(e_{k L}\right)\right), \phi\left(F\left(e_{j U}\right), G\left(e_{k U}\right)\right)\right)
\end{aligned}
$$

Such that

$$
\begin{aligned}
& \varphi\left(F\left(e_{j L}\right), G\left(e_{k L}\right)\right) \\
& =\left\{\frac{\sum_{i}^{n} \max \left\{\min \left(F\left(e_{j L}\right), G\left(e_{k L}\right)\right)\right\}}{\sum_{i}^{n} \max F\left(e_{j L}\right)}, \text { if } F\left(e_{j L}\right)=0, \forall e_{j}\right. \\
& \phi\left(F\left(e_{j U}\right), G\left(e_{k U}\right)\right)=\frac{\sum_{i}^{n} \max \left\{\min \left(F\left(e_{j U}\right), G\left(e_{k U}\right)\right)\right\}}{\sum_{i}^{n} \max F\left(e_{j U}\right)}
\end{aligned}
$$

and 


$$
\gamma=1-\frac{\sum\left|\mu\left(e_{j}\right)-\lambda\left(e_{k}\right)\right|}{\sum\left|\mu\left(e_{j}\right)-\lambda\left(e_{k}\right)\right|}, j, k=1,2, \ldots, n
$$

Definition 5.2. Two GIVFSM's $\mathrm{F}_{\mu}$ and $\mathrm{G}_{\lambda}$ are called significantly similar if $S\left(\mathrm{~F}_{\mu}, \mathrm{G}_{\lambda}\right) \geq 1 / 2$.

Theorem 5.3. Let $F_{\mu}=\left[F\left(e_{j}\right)\left(x_{i}\right), \mu\left(e_{j}\right)\right]_{m \times n}$ and $G_{\lambda}=$ $\left[G\left(e_{k}\right)\left(x_{i}\right), \lambda\left(e_{k}\right)\right]_{m \times n}$ be two GIVFSM's, then

(a) $S\left(F_{\mu}, G_{\lambda}\right) \neq S\left(G_{\lambda}, F_{\mu}\right)$

(b) $H\left(e_{\ell L}\right)\left(x_{i}\right) \geq 0$ and $H\left(e_{\ell U}\right)\left(x_{i}\right) \leq 1$

(c) If $F_{\mu}=G_{\lambda}$, then $S\left(F_{\mu}, G_{\lambda}\right)=1$

Proof:

(a) The proof is straightforward and follows from definition.

(b) From definition, we have

$$
\begin{aligned}
& \varphi\left(F\left(e_{j L}\right), G\left(e_{k L}\right)\right) \\
& =\left\{\frac{\sum_{i}^{n} \max \left\{\min \left(F\left(e_{j L}\right), G\left(e_{k L}\right)\right)\right\}}{\sum_{i}^{n} \max F\left(e_{j L}\right)}, \text { otherwise }\left(e_{j L}\right)=0, \forall e_{j}\right.
\end{aligned}
$$

If $F\left(e_{j L}\right)=0, \forall e_{j}$, then $H\left(e_{\ell L}\right)\left(x_{i}\right)=0$ and if $F\left(e_{j L}\right) \neq$ 0 , for some $e_{j}$, then it is clear that $H\left(e_{\ell L}\right)\left(x_{i}\right) \geq 0$.

Since

$$
\begin{aligned}
& H\left(e_{\ell U}\right)\left(x_{i}\right) \\
& =\max \left(\varphi\left(F\left(e_{j L}\right), G\left(e_{k L}\right)\right), \phi\left(F\left(e_{j U}\right), G\left(e_{k U}\right)\right)\right)
\end{aligned}
$$

Assume that

$\varphi\left(F\left(e_{j L}\right), G\left(e_{k L}\right)\right)=1$ and $\phi\left(F\left(e_{j U}\right), G\left(e_{k U}\right)\right)=1$, then $H\left(e_{\ell U}\right)\left(x_{i}\right)=1$

If $\varphi\left(F\left(e_{j L}\right), G\left(e_{k L}\right)\right)<1$ and $\phi\left(F\left(e_{j U}\right), G\left(e_{k U}\right)\right)<1$, then $H\left(e_{\ell U}\right)\left(x_{i}\right) \leq 1$.

(a) The proof is straightforward and follows from definition.

Theorem 5.4. Let $F_{\mu}=\left[F\left(e_{j}\right)\left(x_{i}\right), \mu\left(e_{j}\right)\right]_{\mathrm{m} \times \mathrm{n}}, G_{\lambda}=$ $\left[G\left(e_{k}\right)\left(x_{i}\right), \lambda\left(e_{k}\right)\right]_{\mathrm{m} \times \mathrm{n}}$ and

$H_{\gamma}=\left[H\left(e_{\ell}\right)\left(x_{i}\right), \gamma\left(e_{\ell}\right)\right]_{\mathrm{m} \times \mathrm{n}}$ be three GIVFSM's, then the following hold:

$$
F_{\mu} \subseteq G_{\lambda} \subseteq H_{\gamma} \Rightarrow S\left(F_{\mu}, H_{\gamma}\right) \leq S\left(G_{\lambda}, H_{\gamma}\right)
$$

Proof: The proof is straightforward and follows from definition.

\section{Conclusion}

We have introduced the concept of GIVFSM's in this paper. Some of its types are defined. Some basic operations like union, intersection, compliment, AND operation and OR operation have been defined and exemplified. Arithmetic mean, geometric mean, harmonic mean and their weighted means are also defined and some properties of these operators are discussed. Furthermore, similarity between two GIVFSM's is discussed. To future concern, GIVFSM's can be used to solve decision making problems in situations where uncertainty involved.

\section{References}

[1] B. V. Gnedenko, "The Theory of Probability", Chelsea, New York, NY, USA, (1962).

[2] L. A. Zadeh, "Fuzzy sets," Information and Computation, vol. 8, pp. 338-353, 1965.

[3] Z. Pawlak, "Rough sets," International Journal of Computer and Information Sciences, 11( 5), 341-356 (1982).

[4] L. A. Zadeh, "The concept of a linguistic variable and its application to approximate reasoning-I," vol. 8, pp. 199-249, 1975.

[5] K. T. Atanassov, "Intuitionistic fuzzy sets," Fuzzy Sets and Systems, vol. 20(1), pp. 87-96, 1986.

[6] D. Molodtsov, "Soft set theory-first results," Computers \& Mathematics with Applications, vol. 37(4-5), pp. 19-31, 1999.

[7] P. K. Maji, R. Biswas, and A. R. Roy, "Soft set theory," Computers \& Mathematics with Applications, vol. 45 (4-5), pp. 555-562, 2003.

[8] M. I. Ali, F. Feng, X. Liu, W. K. Min, and M. Shabir, "On some new operations in soft set theory", Computers \& Mathematics with Applications, vol. 57(9), pp. 1547-1553, 2009.

[9] P. K. Maji, R. Biswas, and A. R. Roy, "Fuzzy soft sets," Journal of Fuzzy Mathematics, vol. 9(3), pp. 589-602, 2001.

[10] A. R. Roy and P. K. Maji, "A fuzzy soft set theoretic approach to decision making problems," Journal of Computational and Applied Mathematics, vol. 203(2), pp. 412-418, 2007.

[11] X. Yang, T. Y. Lin, J. Yang, Y. Li, and D. Yu, "Combination of interval-valued fuzzy set and soft set," Computers \& Mathematics with Applications, vol. 58(3), pp. 521-527, 2009.

[12] Majumdar and S. K. Samanta, "Generalised fuzzy soft sets," Computers \& Mathematics with Applications, vol. 59(4), pp. 425-1432, 2010.

[13] B. K. Saikia, H. Boruah and P. K. Das, "An Applications of Generalized Fuzzy Soft Matrices in Decision Making Problem", IOSR Journal of Mathematics, vol. 10 (1), pp. 3341, 2014.

[14] Shawkat Alkhazaleh, Abdul Razak Salleh, "Generalised Interval-Valued Fuzzy Soft Set", Journal of Applied Mathematics, (2012). doi:10.1155/2012/870504

[15] Mi Jung Son, "Interval-valued Fuzzy Soft Sets", Journal of Korean Institute of Intelligent Systems, vol. 17(4), pp. 557$562,2007$.

[16] P. Rajarajeswari, P. Dhanalakshmi, "Interval-valued fuzzy soft matrix theory", Annals of Pure and Applied Mathematics, vol. 7(2), pp. 61-72, 2014.

[17] Zulqarnain. $M$ and M. Saeed, "An application of interval valued fuzzy soft matrix in decision making", Science International (Lahore), vol. 28(3), pp. 2261-2264, 2016. 
[18] Anjan Mukherjee and Sadhan Sarkar, "Distance based similarity measures for interval-valued intuitionistic fuzzy soft sets and its application", New Trends in Mathematical Sciences, vol. 3(4), pp. 34-42, 2015.

[19] Anjan Mukherjee and Sadhan Sarkar, "Similarity measures for interval-valued intuitionistic fuzzy soft sets and its application in medical diagnosis problem", New Trends in Mathematical Sciences, vol. 2(3), pp.159-165, 2014.

[20] B. Chetia and P. K. Das, "An application of interval valued fuzzy soft sets in medical diagnosis", Int. J. Contemp. Math. Sciences, Vol. 5(38), pp. 1887 - 1894, 2010.

[21] Zhi Xiao Weijie Chen and Lingling Li, "A method based on interval-valued fuzzy soft set for multi-attribute group decision-making problems under uncertain environment" Knowl Inf Syst, vol. 34, pp. 653-669, 2013.

[22] M. Zulqarnain1 and M. Saeed, "A New Decision Making Method on Interval Valued Fuzzy Soft Matrix (IVFSM)", British Journal of Mathematics \& Computer Science, vol.
20(5), pp. 1-17, 2017.

[23] Sarala N. and Prabharathi M., "An application of interval valued fuzzy soft matrix in medical diagnosis" IOSR Journal of Mathematics, vol. 11(1), pp. 01-06, 2015.

[24] Zulqarnain M, Saeed M and Tabasum F., "Comparison between fuzzy soft matrix (FSM) and interval valued fuzzy soft matrix (IVFSM) in decision making", Science International (Lahore), vol. 28(5), pp. 4277-4283, 2016.

[25] Sarala N, Prabharathi M., "An application of interval valued fuzzy soft matrix in decision making problem", International Journal of Mathematics Trends and Technology, vol. 21(1).2015.

[26] Manash Jyoti Borah, Tridiv Jyoti Neog and Dusmata Kumar Sut, "Fuzzy Soft Matrix Theory and its Decision Making", International Journal of Modern Egineering Researh, vol. 2 (2), pp. 121-127, 2012. 